\title{
ASSESSMENT OF PANDEMICS IMPACT ON PUBLIC HEALTH AND AVIATION SECTOR BY USING MATHEMATICAL MODELLING
}

\author{
Peter Szabó, Miroslava Ferencová* \\ Technical University of Košice, Faculty of Aeronautics, Rampová 7, 04121 Košice, Slovakia \\ Eva Szabóová \\ Pavol Jozef Šafarik University in Košice, Ondavská 8, 04011 Košice, Slovakia \\ *Corresponding author. miroslava.ferencova@student.tuke.sk; tel.: +421 903356789
}

\begin{abstract}
The article researches the COVID-19 pandemic impact on the air traffic and aviation sector. The airlines alike the individual countries are introducing the measures intending to stop the COVID-19 pandemic spread. These measures might have a significant impact on the public health of the inhabitants. In the article, the authors assess the viral diseases spread by using mathematical modelling. The first general mathematical model includes the basic epidemiological parameters (basic reproductive number, incubation period, and population). The objective of this model is to explain the basic terms and processes during the transmission of viral diseases. The second mathematical model includes the data analysis of the COVID-19 virus spread in individual countries. The data source for this analysis is the World Health Organization (WHO). A unique protocol to measure viral diseases spread does not exist, hence, the data from this analysis cannot be comparable. Nevertheless, the research results might be useful for public health as well as for the life's segments where the air traffic and airlines belong to.
\end{abstract}

Keywords: mathematical modelling; COVID-19 pandemic; air traffic; online model; regression analysis; basic reproductive number

\section{INTRODUCTION}

Many research activities are interdisciplinary, in a lot of cases even transdisciplinary. To comprehend the connections between them, it is essential to understand the basics and patterns of each research discipline. Our world is accurately described by mathematical models. If we acknowledge it or not, mathematical models construct a background of each research.

In the paper, the authors have jointed mathematical modelling and the spreading of viral diseases; the goal is to evaluate the behavior of the virus spreading so the results can be used for public health service or for the segments where air traffic/airlines belong to. In the second chapter, the authors summarized the information regarding the approach of aviation organizations and airlines towards the current epidemiologic situation as well as the basic terms of the research - basic reproductive number, incubation period, homogeneous population, and collective immunity. Based on the defined terms, the third chapter offers a mathematical model that allows us to model viral diseases spreading for different countries; for modelling, the SageMath System has been used. The calculated basic reproductive numbers for individual European countries based on data from the World Health Organization (WHO) can be found in the fourth chapter. These calculations were done by using regression analysis. Each European country puts different measures to minimize the spreading of viral disease. General findings and results of the analysis are included in the last chapter of this article. 


\section{MATERIALS AND METHODOLOGY}

\subsection{Impact of COVID-19 on aviation sector}

The COVID-19 pandemic has an enormous impact on air traffic, airlines, airports, aircraft operators, etc. A decrease in the number of flights is very significant and notable; during March and April 2020, comparing the operational days with days from last year, this decrease varies between -80 up to $-90 \%$ per day. In the context of this pandemic, EUROCONTROL is publishing a regular comprehensive assessment of the traffic situation in Europe and provides a comparison to the same period in 2019.

At the time of writing this paper, the comprehensive assessment $\left(29^{\text {th }}\right.$ of September) provided the following figures.

The busiest airlines were Ryanair with 1136 flights, followed by Turkish Airlines with 596 flights and EasyJet with 538 flights. The busiest airports were Amsterdam with 615 Dep/Arr flights, followed by Paris/CDG with 553 Dep/Arr flights and Frankfurt (444 Dep/Arr flights).

Intra-Europe flights (flights within the ECAC area) are still at $-54 \%$ compared to 2019 . The most active flows are domestic flows; the highest increase showed flows in Norway $(+7 \%)$, followed by the UK $(+4 \%)$ and France $(+2 \%)$. The busiest nondomestic flows were the flow between the UK and Spain (213 flights, $+2 \%$ ). In the United States, the domestic traffic reached $-50 \%$ of 2019 on the 20th of September while international flights are at $-72 \%$ compared to 2019 (except flows to/from Mexico). In China, the domestic traffic is now close to pre-COVID times (the maximum 12168 flights were reached on 25 August). The number of the international flights is below around $-70 \%$ compared to pre-COVID levels. The domestic and international traffic in the Middle East has been recovering more or less at the same levels.

The results and more details together with the graphs are publicly available and can be accessed via the EUROCONTROL website [0].

The International Civil Aviation Organization (ICAO) published the Economic Impact Analysis Effects of Novel Coronavirus (COVID-19) on Civil Aviation (01 $1^{\text {st }}$ of October). It includes the estimations on world scheduled passenger traffic for the full year 2020. It shows that the overall reduction of seats offered by airlines might be between $50-52 \%$ less; overall reduction of passengers between 2875 to 2978 million less and potential loss of gross operating revenues of airlines between 386 to 399 billion USD. These impacts are dependent on the duration of the outbreak and containment measures, economic conditions, etc. [0].

The diseases such as COVID-19 are putting risk on the public as they can be transmitted between humans easily. However, as assessed by IATA, the risk of catching an infection on the aircraft is lower than catching some infection in indoor environments, such as offices, shopping centers, etc. In modern aircraft, the cabin air is changed more frequently than in offices or shops. For most modern aircraft types, the air supplied to the passenger cabin is either $100 \%$ fresh or is a mixture of fresh and recirculated air that is filtered through HEPA filters of the same efficacy in removing viruses as those used in surgical operating rooms. This has been also shown and proved by 2 studies published by the European Union Aviation Safety Agency (EASA) [0]. The EASA studies provide information about cabin air quality on board of aircraft.

However, it is not only time on the board of the aircraft that we spend during travelling; we need also to consider the time spent on the airport terminals, etc. As this kind of disease can spread internationally very easily, ICAO has established the appropriate measures in the domain of air travel that can contain and/or detect such spread. ICAO holds an extensive body of guidance material and operates a robust regulatory framework that is currently helping its Member States to deal with COVID-19. ICAO together with other involved stakeholders developed a single source for aviation specific guidelines to assure the appropriate planning and actions at all levels to mitigate the effect of a human break. For instance, ICAO Standards require a National Aviation Plan to be in place. It should follow the guidance provided by ICAO and preparedness guidance available from the World Health Organization (WHO). In addition, as per ICAO Doc10042 Model National Air Transport Facilitation Programme, a National Air Transport Facilitation Programme or similar body must also be in place to 
clarify the roles and responsibilities of all relevant government agencies and ministries and other stakeholders for the prevention of the spread of disease. States are obliged to establish National Air Transport Facilitation Committees to coordinate broad policy issues including responses to public health emergencies of international concern.

IATA also recommends that all air carriers have emergency response plans to deal with public health emergencies. A part of this plan involves the guidelines and practices for airline staff in the event of public health emergencies. These plans have been developed based on experience with different outbreaks, such as measles or ebola [0].

On 21 May 2020, EASA and the European Centre for Disease Prevention and Control (ECDC) have developed the 'COVID-19 Aviation Health Safety Protocol'. The protocol has been developed with a purpose to maintain safe and secure operations whilst minimizing the risk of virus transmission. It serves as an aviation health safety protocol and provides a source of best practice on how airport operators, aircraft operators, and national aviation authorities can ensure the health and safety of passengers, as well as the staff and crew who serve them. The guidelines provide preventive measures for passengers and the crew member's management. For passengers, there are various measures in place. At all times passengers should be reminded about hand hygiene, maintaining the social distance, wearing the medical face masks, etc. The aircraft operators in cooperation with airport operators should inform passengers of the travel restrictions for any passenger who may have COVID-19 compatible symptoms before arriving at the departure airport. To reduce the risk of transmission of the virus from potential asymptomatic contagious passengers, several considerations are in place, such as cleaning and disinfection, thermal screening at the departure airport, protective screens at the check-in desks, passport controls, and information counters. When already in an aircraft, the flight crew should provide guidance material to passengers on how to correctly apply the preventive measures (hand hygiene, appropriate use of face masks, respiratory etiquette, etc.). Additionally, aircraft operators should also consider reducing food and drink service on board, stop duty-free, or other non-essential product sales on board, avoid cash payments as much as possible. If after take-off, a passenger shows symptoms compatible with COVID-19, a passenger should be wearing a face mask and should be isolated immediately; consequently, the flight crew should inform the destination airport via the air traffic control system and follow their instructions (more detailed measures are part of the protocol). To reduce the risk of virus transmission at the airport after the arrival, special measures should be considered for the management of arriving and transiting passengers as well, e.g. physical distancing as much as possible during the disembarkation procedure, passenger locator card, thermal screening at the arrival airport, etc.

Crew members, airport staff members, and service supplier staff members should be exempt from the airport's COVID-19 screening procedures. Additional operational measures should be considered following the EASA Guidelines for the management of crew members [0].

\subsection{Basic concepts of epidemiology}

The mathematical model includes the terms used in epidemiology, therefore, there will be introduced in this subsection. The definitions are simple and serve only the purposes of the research. The basic reproductive number is the average number of people infected from one to another person. This number refers to the estimated contagiousness of an infectious person. It is the average number of people 1 infectious person is expected to infect in an entirely susceptible population (= population where there is no immunity or vaccination). If this number is between $2-4$, it means that in an entirely susceptible population, 1 infected individual will, on average, infect 2-4 other people in the absence of control measures (social distancing, hand hygiene...) [0].

The incubation period is the time elapsed between exposure to a pathogenic organism and onset of clinical symptoms and when signs are first apparent. The population is all inhabitants who live in a particular state or geographical area. 


\section{RESULTS}

\subsection{Mathematical model of spreading the viral diseases}

The mathematical model is based on the basic parameters defined in subsection 0 . The main rule of epidemiology expresses that to control an epidemic, the basic reproductive number needs to be less than 1 and so, the total number of infected people declines and an epidemic ceases to exist.

By default, the values of the basic parameters for the COVID-19 in Slovakia are set as follows:

- the basic reproductive number value is set to 2.2 (infected people)

- the incubation period is set to 6.4 (days)

- the population is set to 5400000 (inhabitants)

These parameters can be adjusted for each country as necessary; different scenarios can be created by setting different values for the basic reproductive number, incubation period, and population.

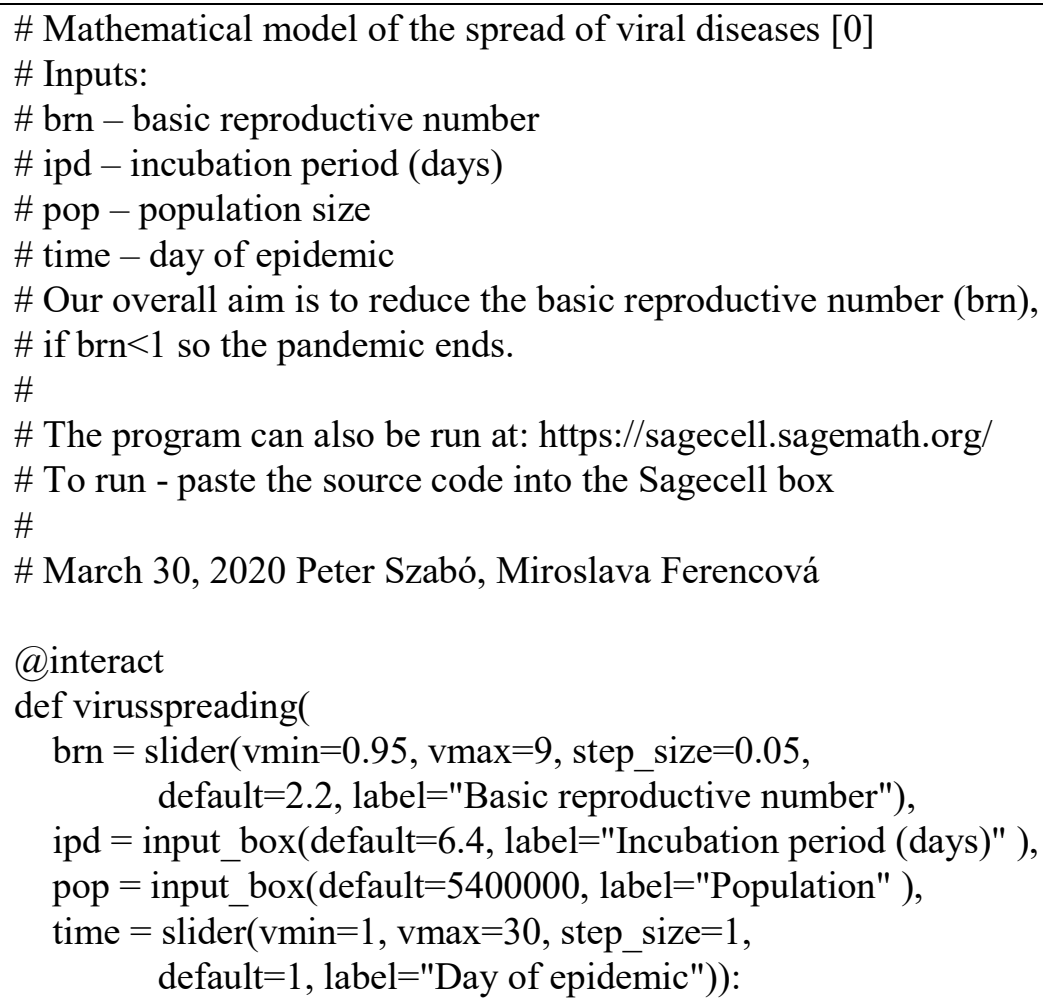


print ("Day of epidemic

:",time)

print ("Number of infected individuals :",round(f(time)))

print ("Size of population : :"pop)

if brn>1: print ("The whole population can be infected in: ",round(days)-time, "days")

else: print ("End of epidemic")

Once the program is activated, there is a possibility to adjust a day of the epidemic and thereafter, to model the estimated situations. The models of these situations will represent a time when there are no control measures applied and so the virus is spread rapidly.

In the opposite case, i.e. an epidemic is controlled, then by putting the regulations (e.g. isolation of infected people from the remaining population), we can achieve a slowdown of the virus spread together with a decrease in the basic reproductive number. Figure 1 represents the online mathematical model that can be run and used online.

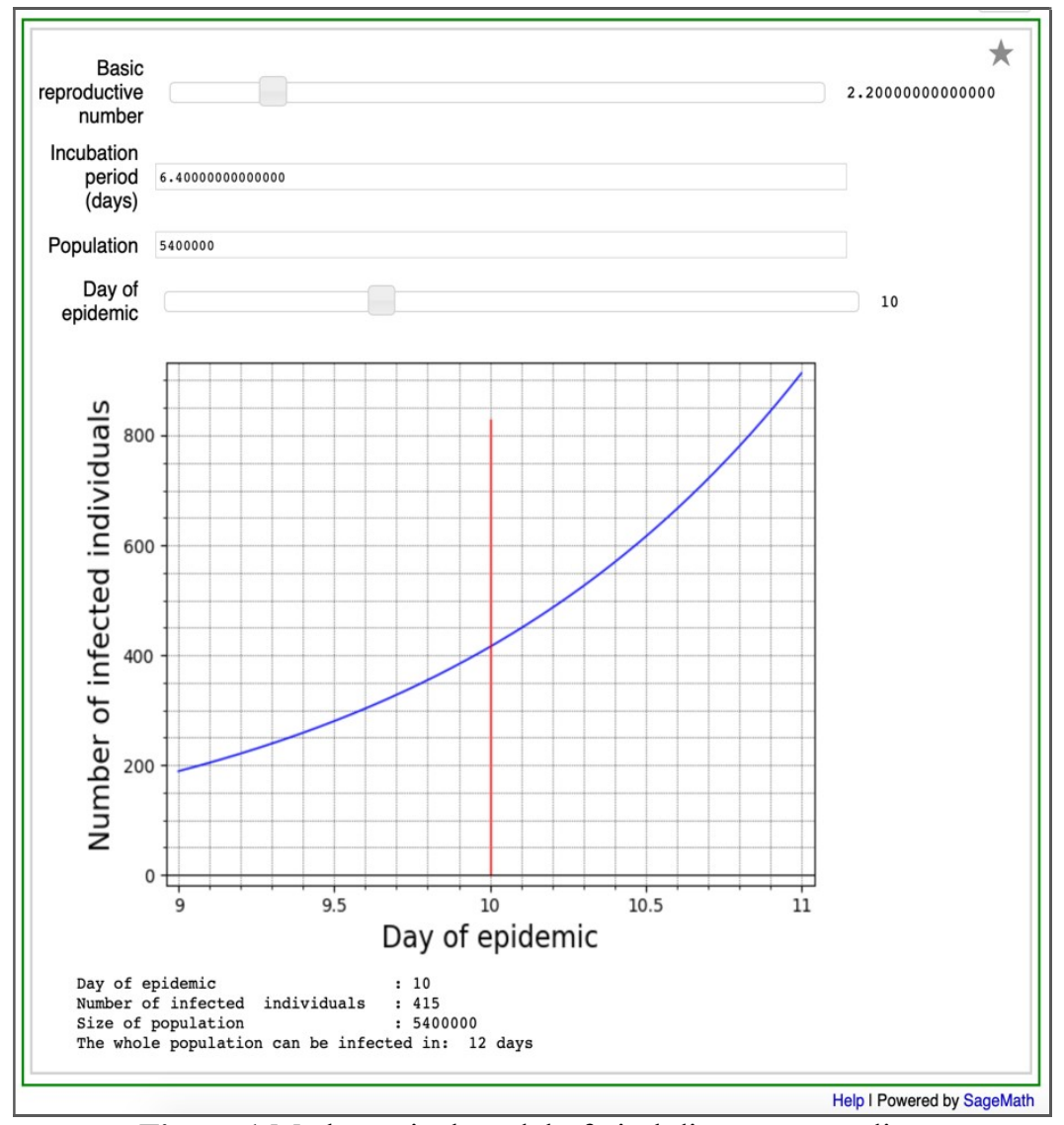

Figure 1 Mathematical model of viral diseases spreading

\subsection{Data analysis and the calculations of basic reproductive number}

Based on the data published on the WHO website, the next part of the research is dedicated to the investigation of how the virus is spread in individual countries. Daily, each country provides information about the new cases positively tested to COVID-19 together with the number of deaths in consequence of this virus.

At the time of writing this paper, there was no unique protocol in place that would determine which cases should be reported and included in the database linked with this virus. And that is the reason why the results might differ. 


\subsubsection{Methodology and research goals}

The authors' goal was to calculate and compare the basic reproductive number for each country in the initial phase of the virus spreading. The initial phase is declared as the first week in which the country recorded the existence of the virus.

During the next 6 weeks, the aggregate numbers of infected people were calculated weekly with the following input data:

Country: Portugal

Period: 3.3.2020-21.4.2020

Aggregate numbers of infected in the first 6 weeks (based on the WHO data):

$$
\text { [(0,37), (1,329), (2,2058), (3,6406), (4,11728), (5,16932), (6,20861)] }
$$

Based on the input data (country, time period, the beginning of the pandemic spread, weekly aggregate numbers of the infected in the first 6 weeks), the authors aimed to estimate the basic reproductive number; it is the lower estimate of this number because the input data might not cover all infected people in the country.

The function of the pandemic spread is defined by the formula:

$$
f(x)=a^{x} / b
$$

where: $x$ represents a day of the pandemic, $a$ is the basic reproductive number, and $b$ is the incubation period (in days).

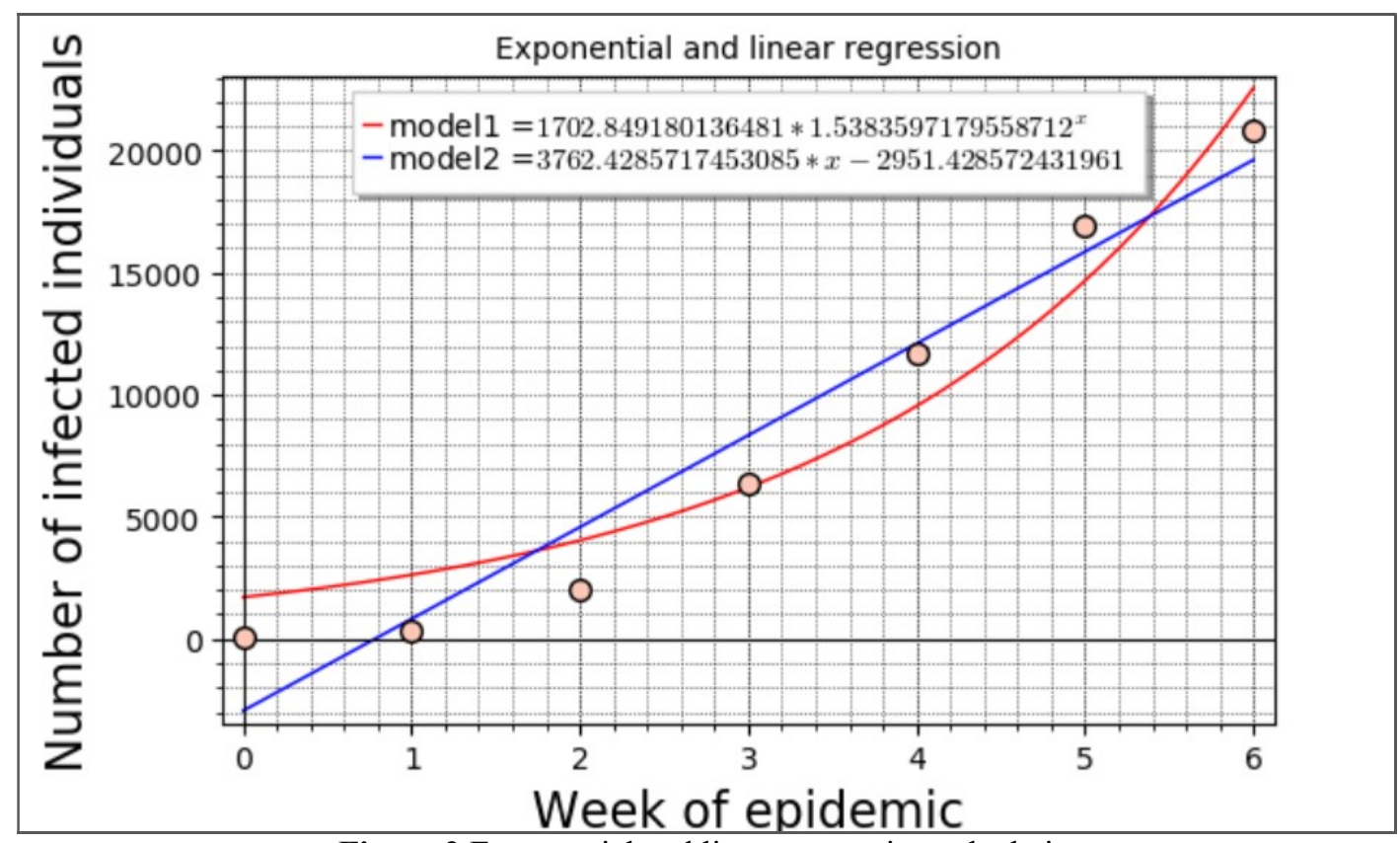

Figure 2 Exponential and linear regression calculation

The goal is to determine the number $a$ based on the input data; number $b$ is a constant (the incubation period is the same for this type of virus), $\mathrm{b}=6,4$. To determine the number $a$, an exponential regression was applied; this method will return the exponential function which is the closest to the input points. Consequently, the linear regression line was calculated. In the second model, authors anticipated that the reproductive number $a=1$ which is not the case; that is why this 
model cannot be used. In the first model, the base of the exponential function will be an estimation of the searched reproductive number. Figure 2 offers the results of the calculation.

\section{DISCUSSION}

The estimate of the reproductive number is $a=1,53$. In the linear model, the function $f(x)=k x+q$ was received; in the function, the number $k$ characterizes a weekly increase in the number of infected cases. The model of the exponential and linear regression was applied in the selected countries and the following table created:

Table 1 Exponential and linear regression - comparison

\begin{tabular}{|c|c|c|c|}
\hline Country & $\mathrm{k}$ & brn (a) & ipn (b) \\
\hline Spain & 28588 & 1,68 & 7496 \\
\hline Italy & 23272 & 1,62 & 7669 \\
\hline China & 16788 & 1,26 & 26717 \\
\hline Germany & 14376 & 1,8 & 2611 \\
\hline Russia & 6561 & 2,67 & 130 \\
\hline UK & 6557 & 1,7 & 1634 \\
\hline Portugal & 3566 & 1,57 & 1380 \\
\hline Austria & 2678 & 1,5 & 1351 \\
\hline Poland & 2581 & 1,57 & 1004 \\
\hline Czechia & 1240 & 1,45 & 775 \\
\hline Ukraine & 800 & 2,28 & 38 \\
\hline Hungary & 324 & 1,68 & 89 \\
\hline Greece & 314 & 1,57 & 127 \\
\hline Slovenia & 232 & 1,35 & 232 \\
\hline Slovakia & 175 & 1,59 & 68 \\
\hline France & 85 & 6,5 & 0,0088 \\
\hline USA & 51 & 5,53 & 0,015 \\
\hline Canada & 7 & 2,38 & 0,305 \\
\hline
\end{tabular}

The countries in the table are ordered by the weekly increase in the amount of infected people by COVID-19 in the first 6 weeks. In the second column, the authors estimated the basic reproductive number for each country. This number is also dependent on the number of conducted tests as well as the testing methodology. Assuming that the incubation period is $b=6,4$, then in the third column, the constant amounts $1 / 6,4=0,15625$ should be received. Now, the objective of applied mathematics is to find the transformation formula to calculate a comparative estimate of the second column with values of the basic reproductive number. The incubation period amount should be assigned by epidemiologists; the value of the incubation period is not a fixed value, but it is determined by the interval [idb $\left.\mathrm{in}_{\min }, \mathrm{idb}_{\max }\right]$.

\section{CONCLUSION}

The article joints 2 research disciplines - mathematical modelling and the public health sector. By creating a mathematical model in the SageMath system and using regression analysis, the authors calculated the basic reproductive numbers based on the data from WHO and assessed the spreading of the viral diseases in different countries. In addition to that, the article also provides the impact of COVID-19 pandemic on the aviation sector and the approach of the individual organizations towards this pandemic. 


\section{References}

[1] EUROCONTROL. Comprehensive Assessment of COVID-19's Impact on European Air Traffic. Available at: https://www.eurocontrol.int/publication/eurocontrol-comprehensive-assessmentcovid-19s-impact-european-air-traffic.

[2] ICAO (International Civil Aviation Organization). Effects of novel Coronavirus (COVID-19) on Civil Aviation: Economic Impact Analysis. Available at: https://www.icao.int/sustainability/Doc uments/COVID-19/ICAO_Coronavirus_Econ_Impact.pdf

[3] EASA (European Union Safety Agency). Studies on cabin air quality. Available online: https://www.easa.europa.eu/newsroom-and-events/news/easa-publishes-two-studies-cabin-air-quality

[4] IATA (International Air Transport Association). Air Transport \& COVID-19 Coronavirus. Available online: https://www.iata.org/en/programs/safety/health/diseases/\#tab-1

[5] EASA (European Union Safety Agency). COVID-19 Aviation Health Safety Protocol. Available at: https://www.easa.europa.eu/newsroom-and-events/press-releases/easaecdc-issue-jointguidelines-assure-health-safety-air-travel

[6] Field Epidemiology Manual (FEM) Wiki. Introduction to Public Health and basic concepts. Available at: https://wiki.ecdc.europa.eu/fem/Pages/Introduction $\% 20$ to $\% 20$ Public $\% 20 \mathrm{Health} \% 20$ and $\% 20$ basic\%20concepts.aspx

[7] Szabó, P.; Galanda, J. Sage math for education and research. 15th International Conference on Emerging eLearning Technologies and Applications (ICETA), 2017, (pp. 1-4). IEEE. DOI: 10.1109/ICETA.2017.8102535

[8] E-course (Online model). Sagemath: Modelling the spread of viral diseases. Available online: http://people.tuke.sk/peter.szabo/SageCell_Covid_EN.html

[9] ICAO (International Civil Aviation Organization). Aviation and COVID-19. Available at: https://www.icao.int/Security/COVID-19/Pages/default.aspx

Received 10, 2020, accepted 12, 2020

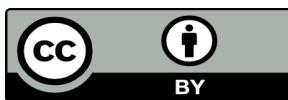

Article is licensed under a Creative Commons Attribution 4.0 International License 